\title{
Optical Properties of a Low Birefringence Polyester Containing Fluorene Side Chain I
}

\author{
Kazuo SAKURAI $^{\dagger}$ and Michiaki FUJI \\ O-PET Development, Kanebo Ltd., \\ 5-90, Tomobuchi, Miyakojima, Osaka, 534-8666, Japan
}

(Received January 14, 2000)

\begin{abstract}
We examined optical properties of a new polyester resin synthesized from 9,9-bis[4-(2-hydroxy-ethoxy)phenyl]fluorene (BPEF), ethylene glycol, and dimethyl terephthalate (DMT) by a melt-condensation polymerization. When the mol ratio of BPEF to the total diol $(k)$ was increased above 0.2 , the refractive index exceeded 1.6 . From rheooptical measurements carried out below the glass transition temperature, the intrinsic birefringence was found to decrease with increasing $k$. For the sample with $k=0.7$, the intrinsic birefringence was as small as 0.07 , about one third the value commonly observed for poly(ethylene terephthalate). Molecular modeling of the BPEF unit indicates that the fluorene side chain is perpendicularly aligned to the main chain and this stereo structure appears responsible for the small birefringence of the polyester studied.
\end{abstract}

KEY WORDS Polyester/Fluorene / Intrinsic Birefringence / High Refractive Index /

Some amorphous and glassy polymers are molded into optical devices such as lens, optical fibers, and optical disks. However, when optical isotropy is required, the birefringence $(\Delta n)$ of the polymers becomes a serious disadvantage. The birefringence of molded plastics is generated by the frozen resin-flow formed when injectionmolded. This flow is unavoidable even if the molding is done with great care. One approache to reduce $\Delta n$ is therefore to lower the intrinsic birefringence $\left(\Delta n_{0}\right)$ of the polymer by controlling its chemical structure ${ }^{1}$ and that can be made, for example, by use of olefin or acrylate units. $^{2}$ However, the refractive indices $(n)$ of polyolefins and polyacrylates are usually low, appearing around 1.5, and this fact sometimes may make them less attractive for use as optical material. Polymer's $n$ is essentially proportional to the density and the way to increase $n$ would be to incorporate aromatic rings. ${ }^{2}$ Aromatic rings, however, have relatively large birefringence, so that it is necessary for acquiring both, high refractive index and low intrinsic birefringence, to arrange aromatic rings spatially so as to minimize their optical anisotropy. Based on this idea, Fuji et al. ${ }^{3}$ introduced a fluorene moiety into the poly(ethylene terephthalate) (PET) unit and found drastic decrease in $\Delta n$. This paper examines optical properties of this series of copolyesters, especially focusing on evaluation of $\Delta n_{\mathrm{o}}$ below the glass transition temperature $\left(T_{\mathrm{g}}\right)$.

For glassy materials, $\Delta n$ is related to the stress $(\sigma)$ by $^{4}$

$$
\Delta n=C_{\mathrm{p}} \sigma
$$

where $C_{\mathrm{p}}$ is the photoelastic coefficient. For polymers, $C_{\mathrm{p}}$ depends on time, so that it cannot be treated as a material constant. Inoue and $\mathrm{Ozaki}^{5}$ extensively studied the dynamic (i.e., time-dependent) birefringence in the glass-to-rubber transition zone (i.e., above $T_{\mathrm{g}}$ ) and interpreted its high frequency behavior in terms of the physics of the glassy state. According to them, $\Delta n$ in the glassy state is determined by the chain orientation and the rotational orientation of monomer units. However, since the temperature-time superposition may not hold below $T_{\mathrm{g}}$, their approach based on it is not always adequate to explore the nature of the glassy polymers.

\section{EVALUATION OF THE INTRINSIC BIREFRINGENCE IN THE GLASSY STATE}

Fujita et al. ${ }^{6}$ who measured $\Delta n$ for some amorphous polymers uniaxially elongated below $T_{\mathrm{g}}$, proposed that the birefringence in the glassy state can be separated into two components, one associated with the chain orientation and the other, with the origin of elasticity in glassy materials. Figure 1 illustrates their procedure of data analysis. In the linear elastic region usually found at the stretch ratio $(\lambda)<1 \%, \Delta n$ and $\sigma$ are plotted against $\lambda$ and the values of $\Delta n$ and $\sigma$ are read off at a certain fixed $\lambda\left(\lambda_{\mathrm{i}}\right.$ in the figure). Both $\Delta n$ and $\sigma$ thus determined decrease with increasing temperature and when $\Delta n$ at different temperatures are plotted against the corresponding $\sigma$ (the panel $\mathrm{C}$ ), the data points follow a straight line. According to Fujita et al., the slope of the line is independent of $\lambda$, and the intercept is proportional to $\lambda_{\mathrm{i}}$. This finding led them to an empirical expression for $\Delta n(\lambda)$ at sufficiently small $\lambda$ :

$$
\Delta n=\Delta n_{\text {or }}+C_{\mathrm{e}} \sigma
$$

where $\Delta n_{\mathrm{or}}$ is proportional to $\lambda$, and $C_{\mathrm{e}}$ is independent of $\lambda$.

Birefringence can be attributed to the preferential orientation of dipole moments. For polymeric glasses, this orientation is induced by the cooperative movement of the neighboring structural units (local orientation) as well as the deformation of the overall chain conformation (chain orientation). ${ }^{7,8}$ Thus, Fujita et al. attempted to interpret eq 2 by assuming that the local orientation

\footnotetext{
${ }^{\dagger}$ To whom correspondence should be addressed. Present address: Chemotransfiguration Project, Japan Science and Technology Corporation (JST), 2432 Aikawa, Kurume, Fukuoka 839-0861 Japan.
} 

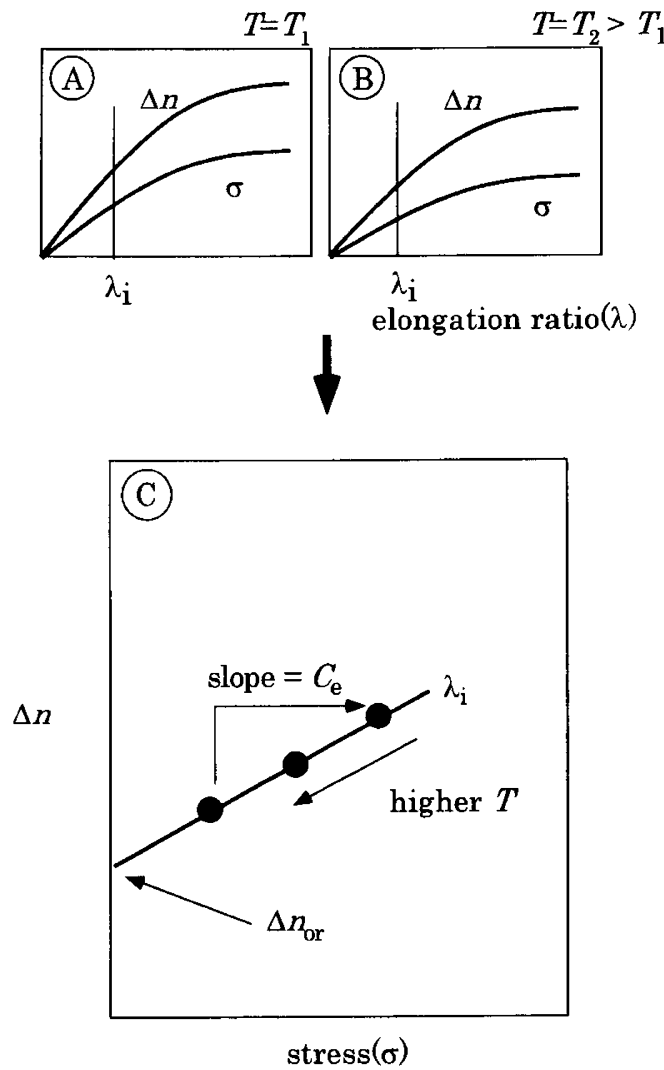

Figure 1. Schematic diagram to illustrate Fujita's method. The birefringence $(\Delta n)$ and stress $(\sigma)$ are measured at various temperatures $\left(T_{1}\right.$ and $T_{2}$ ) below the glass transition temperature. From the data obtained, $\Delta n$ is plotted against $\sigma$ at a certain elongation ratio $\left(\lambda=\lambda_{\mathrm{i}}\right)$, and $\Delta n_{\mathrm{or}}$ and $C_{\mathrm{e}}$ in eq 2 are evaluated from the intercept and slope of the plot.

is the major origin of the stress in the glass state ${ }^{8}$, so that the second term in eq 2 describes its contribution to the birefringence. The intercept $\Delta n_{\text {or }}$ is then the birefringence of a hypothetical elongated sample in which no local orientation occurs and hence $\sigma=0$. Therefore, it is reasonable to assume, as Fujita et al. did, that $\Delta n_{\text {or }}$ follows the same relation as the birefringence for the rubbery state,

$$
\Delta n_{\mathrm{or}}=f \Delta n_{\mathrm{o}}
$$

where $\Delta n_{\mathrm{o}}$ is the intrinsic birefringence and $f$ is the orientation factor. This equation allows $\Delta n_{0}$ to be calculated from $\Delta n_{\text {or }}$, since $f$ for the rubbery state may be approximated by the well-known relation of $f=(3 / 5) \lambda .{ }^{9}$

The above analysis requires that the following conditions be satisfied; (1) $\lambda$ is in the linear elastic region where $\Delta n$ and $\sigma$ linearly increase with $\lambda$ and neither relaxation nor yielding occurs, (2) the stress due to the conformational entropy (i.e., due to the chain orientation) plays a negligible role in the observed stress, and (3) $f$ depends only on $\lambda$. Condition (1) is well obeyed if $\lambda$ is chosen sufficiently small. Condition (2) holds in the glass state, since the rubbery modulus usually being the order of $10^{4}-10^{6}$ dyne $\mathrm{cm}^{-2}$ is smaller by a few orders of magnitude than the observed glassy moduli. ${ }^{8}$ Condition (3) is acceptable because for $\lambda<1 \%$ the affine deformation

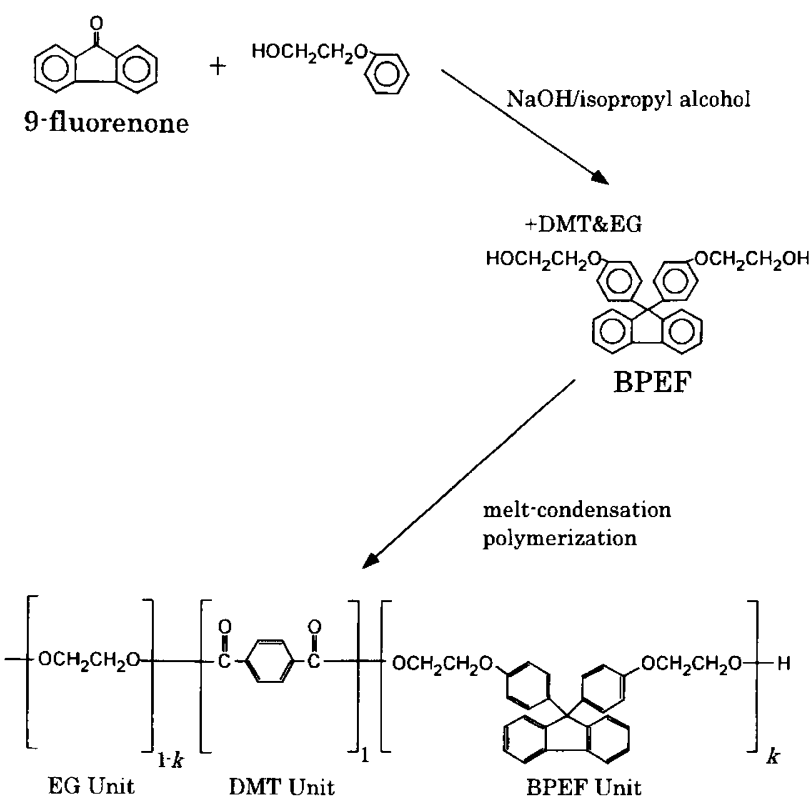

Figure 2. Synthetic procedure of 9,9-bis[4-(2-hydroxy-ethoxy)phenyllfluorene (BPEF) and the polyester containing the BPEF unit.

may hold in good approximation. ${ }^{9}$

Fujita et al. applied their method to $\Delta n$ for polystyrene, polycarbonate $A$, and poly(phenylene oxide) and successfully analyzed the birefringence data below $T_{\mathrm{g}}{ }^{6}$ In particular, the $\Delta n_{0}$ values evaluated coincided with those obtained in the rubbery state. ${ }^{6}$ Their original paper $^{6}$ makes no mention of the physical meaning of $C_{\mathrm{e}}$. It is important that this coefficient is different from the photoelestic coefficient defined by eq 1 . Recently, Inoue and Ozaki ${ }^{10}$ showed that the method they proposed is identical with Fujita's and that $C_{\mathrm{e}}$ is equivalent to the coefficient that they introduced to describe the monomer orientation around the chain axis.

\section{EXPERIMENTAL}

\section{Synthesis}

We carried out copolymerization of 9,9-bis[4-(2hydroxy-ethoxy) phenyllfluorene (BPEF), ethylene glycol(EG), and dimethyl terephthalate (DMT) at a reaction temperature of $270-300^{\circ} \mathrm{C}$ using conventional meltcondensation technique $\mathrm{e}^{11,12}$. The copolymer ratio was controlled by changing the BPEF/DMT molar ratio from 0.05 to 0.95 . For all fed ratios, the mixture of the reactants was homogeneous throughout the polymerization, suggesting the occurrence of random copolymerization. BPEF was prepared by coupling 9-fluorenone with 2phenoxy ethanol and purified by recrystallization in methanol. ${ }^{13}$ DMTA and EG were used as received from Wako Chemical. Figure 2 illustrates the synthetic procedures for BPEF and the polyester described above. Table I presents the sample codes and the fed ratios as well as the results of molecular characterizations described below. The two numbers following $\mathrm{TF}$ in the codes give the $\mathrm{BPEF} / \mathrm{EG}$ fed ratios. 
Table I. Sample code, feed, composition, and some molecular characterristics

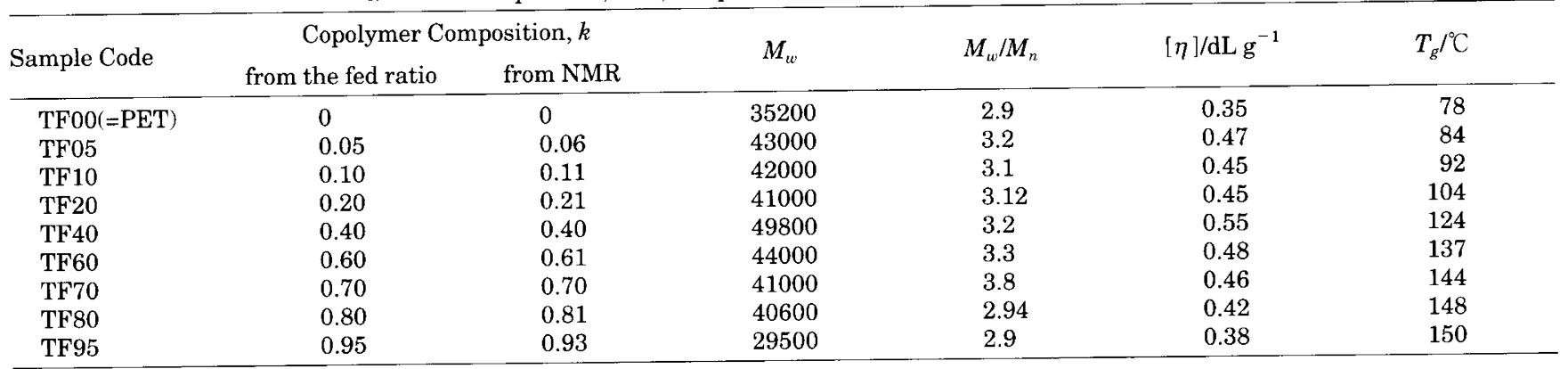

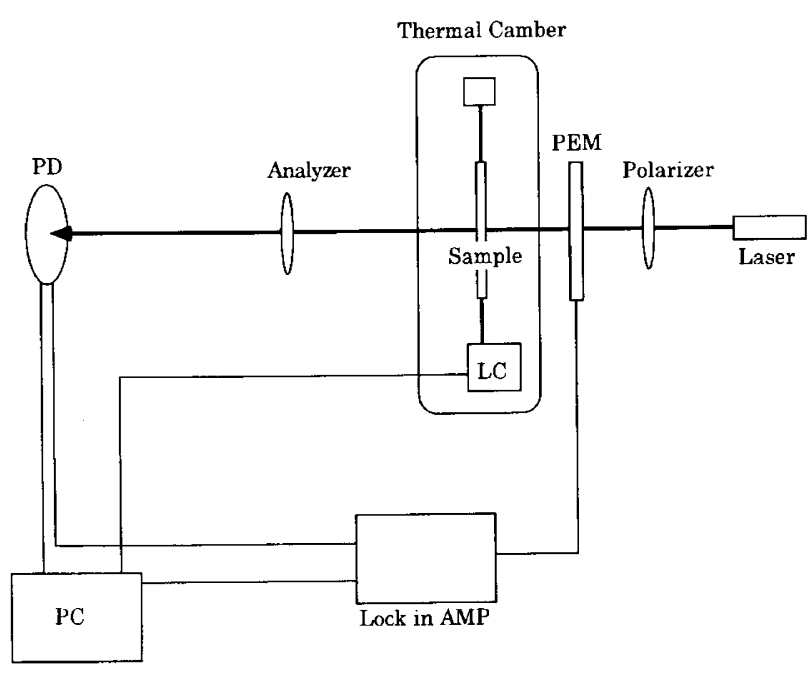

Figure 3. The apparatus for rheo-optical measurement: PMA, photo elastic modulator; PD, photodetector; LC, load cell; PC, computer; AMP, amplifier.

\section{Material Characterization}

Gel permeation chromatograms (GPC) for all samples were obtained with a Toyo Soda HCL-802 A calibrated with standard narrow-distribution polystyrene samples, and the weight average molecular weights $\left(M_{w}\right)$ and the polydispersity indices $\left(M_{w} / M_{n}\right)$ were calculated from them by the conventional methods, where $M_{n}$ is the number average molecular weight. The intrinsic viscosity $([\eta])$ was measured in a mixture of $1,1,2,2$,tetrachloroethane and phenol $\left(4: 6\right.$ in weight) at $25^{\circ} \mathrm{C}$ by a Ubbelohde capillary viscometer. ${ }^{1} \mathrm{H}$ NMR measurements at $300 \mathrm{MHz}$ were made for all samples of about 10 wt\% concentration in $\mathrm{CDCl}_{3}$ at room temperature with a Brucker AM 300 spectrometer to determine the copolymer composition. The refractive index and Abbe's number were measured with an Abbe refractometer (Atago DR-M 2). Heating thermograms at a rate of $10^{\circ} \mathrm{C} \mathrm{min}-1$ were obtained using a Seiko differential scanning calorimeter type 220 . The transmittance was measured for the TF 40 and TF 70 samples $2 \mathrm{~mm}$ thick using a $\mathrm{Hi}$ tachi U-3200 spectrometer.

\section{Birefringence and Stress Measurement}

Figure 3 shows a schematic diagram of our apparatus to measure $\Delta n$ and $\sigma$ for an elongated sample. In a ther- mally controlled camber, a sample film about $200 \mu \mathrm{m}$ thick and $2 \mathrm{~mm}$ wide was stretched from both sides of it at an elongation rate of $1 \% \mathrm{~s}^{-1}$. The optical system consisted of a helium-neon gas laser, a polarizer, a photoelastic modulator, an analyzer, and a photo detector. The signal from the photo detector was analyzed with a lock-in amplifier and accumulated as a function of time by a computer.

Not being amorphous (see molecular characterization section), the samples TF00 and TF05 were excluded from the rheo-optical measurements. The other seven samples were first annealed above $T_{\mathrm{g}}+10^{\circ} \mathrm{C}$ for a period of about $100 \mathrm{~min}$, and subjected to the measurement of $\Delta n$ and $\sigma$ in the temperature range from $23^{\circ} \mathrm{C}$ to $T_{\mathrm{g}}+5^{\circ} \mathrm{C}$ at $\lambda<3 \%$ and an elongation rate of $1 \% \mathrm{~s}^{-1}$. For some samples, the measurement was repeated by changing the elongation rate from 0.1 to $20 \% \mathrm{~s}^{-1}$. In most cases the linearity between $\Delta n$ and $\sigma$ failed to hold at $\lambda$ beyond $1-1.5 \%$, and hence the data for $\lambda<1 \%$ were used for the analysis.

\section{Molecular Modeling}

The molecular structure of BPEF with the minimum structural energy was sought by a structural energy minimization subroutine in Chem $3 \mathrm{D}$, and the structural data obtained were inputted into a semi-empirical molecular orbital program MOPAC to calculate the polarizability tensor. The resulting tensor was transformed into a diagonal matrix and the intrinsic birefringence of the BPEF unit was calculated. ${ }^{14}$ In the same way, the intrinsic birefringence of the DMTA unit was calculated.

\section{RESULTS}

\section{Molecular Characterization}

Figure 4 shows the ${ }^{1} \mathrm{H}$ NMR spectrum for TF40, which is typical of the polyesters studied. The spectrum exhibits aromatic peaks around $8.2 \mathrm{ppm}$ due to DMT, those at $6.9,7.2-7.5$, and $7.8 \mathrm{ppm}$ due to $\mathrm{BPEF}$, methylene peaks at $4.8 \mathrm{ppm}$ in the EG unit, and those at 4.7 and 4.4 ppm in the BPEF unit. The ratio of the integrated methylene peaks for $\operatorname{EG}(4.8 \mathrm{ppm})$ and $\operatorname{BPEF}(4.7$ and $4.4 \mathrm{ppm})$ was used to determine the copolymer composition (i.e., $k$ in Figure 2), and the results are presented in Table I. The $k$ values thus determined come close to those calculated from the fed ratios. Table I also presents $M_{w}, M_{w} /$ $M_{n}$, and $[\eta]$ for all samples studied.

Figure 5 compares DSC heating thermograms for seven samples. For TF05 the melt-crystallization and 


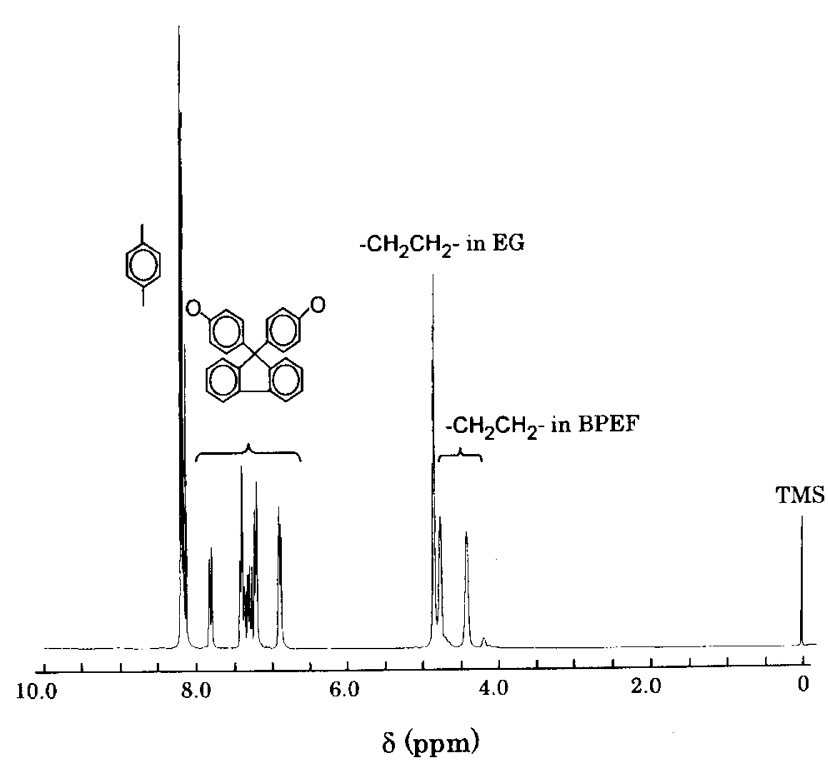

Figure 4. ${ }^{1} \mathrm{H}$ NMR spectrum for the polyester TF40 $(k=0.4)$.

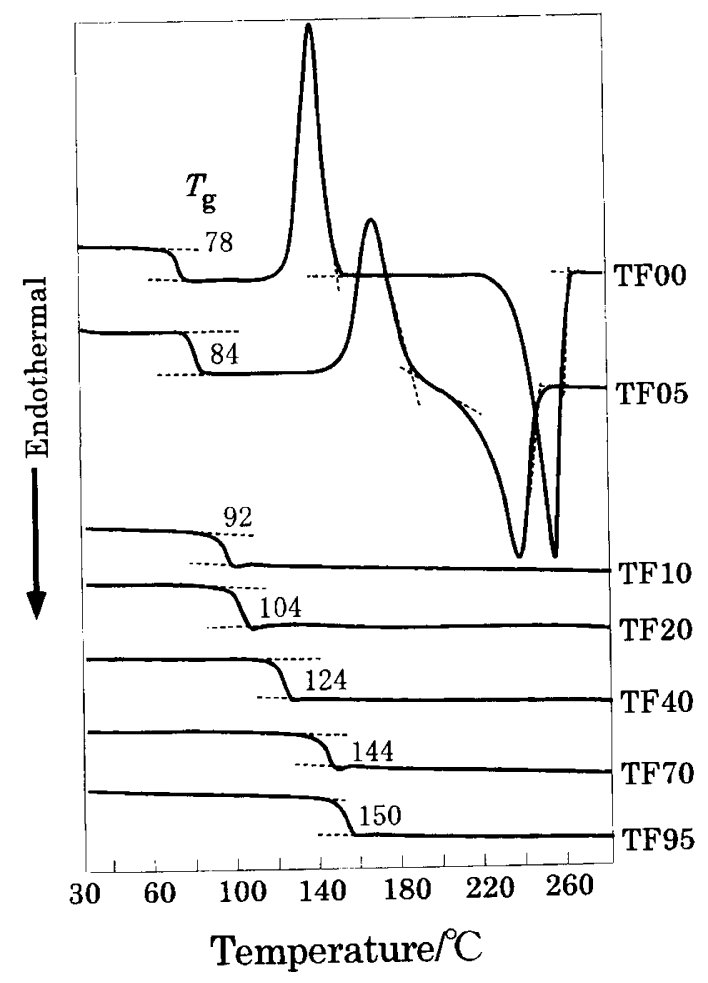

Figure 5. Comparison of DSC thermograms.

melting peaks for the PET moiety are observed above $T_{\mathrm{g}}$. As is generally the case for copolymers, the onset temperature shifts higher for the melt-crystallization and lower for the melting than that for pure PET (i.e., TF00). The samples with $k \geq 0.1$ show no crystallization peak but only the glass transition, so that they can be regarded as amorphous. $T_{\mathrm{g}}$ increases with increasing $k$ and reaches $150^{\circ} \mathrm{C}$ at $k=0.95$, one of the highest $T_{\mathrm{g}}$ for amorphous polyesters. ${ }^{15}$ Figure 6 plots $1 / T_{\mathrm{g}}$ against $\psi$,

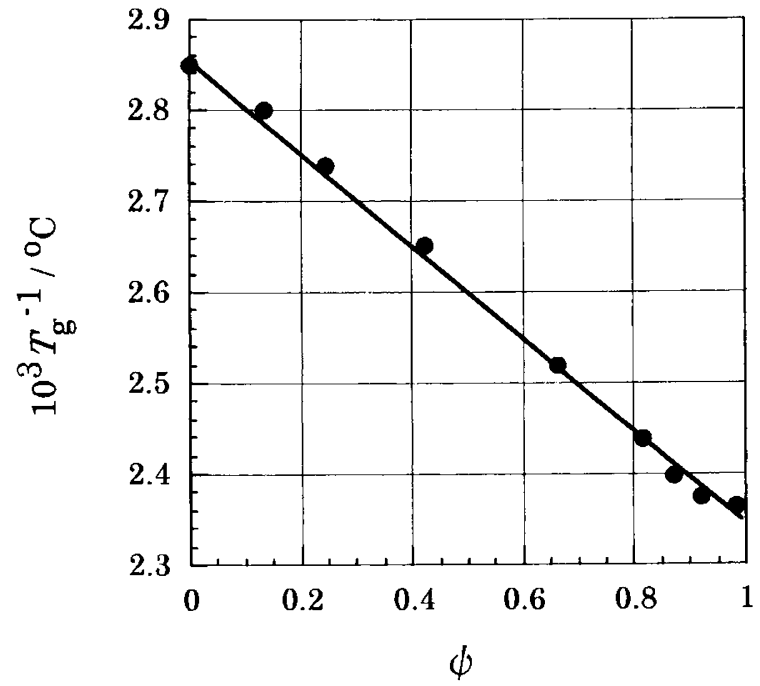

Figure 6. The Fox plot for the composition dependence of glass transition temperatures.

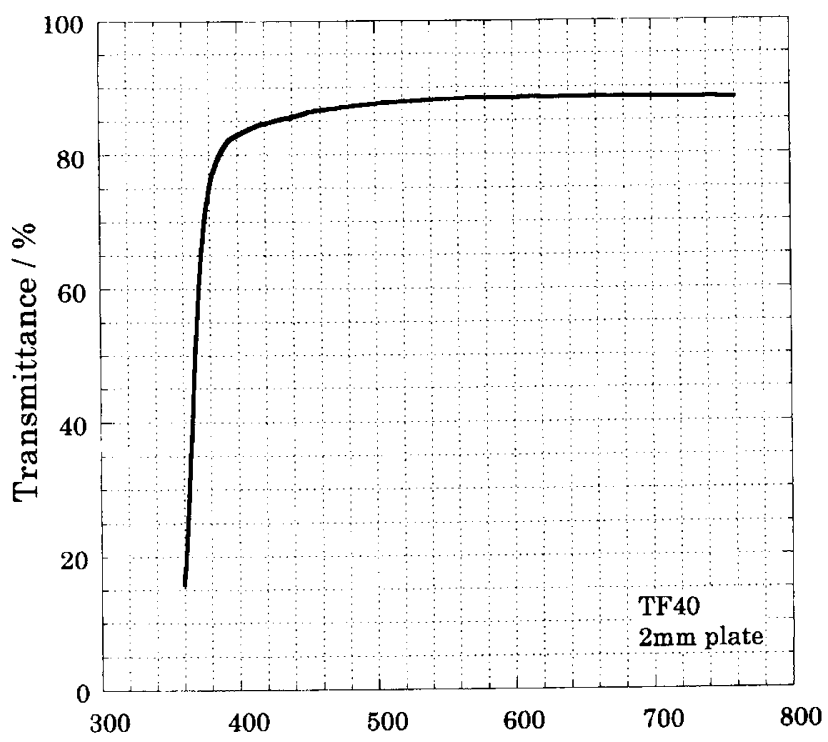

Wavelength / nm

Figure 7. The transmittance spectrum for the TF 40 plate $2 \mathrm{~mm}$ thick.

where $\psi$ is the volume fraction of BPEF and the DMTA bonded to $\mathrm{BPEF}$ in the polyester and calculated by assuming that the specific volumes of the EG, DMTA, and $\mathrm{BPEF}$ units are equal to those of the respective monomers. The data points in the figure can be fitted by a straight line, indicating that the Fox relation is valid, therefore substantiating the copolymerization to proceed randomly. ${ }^{16}$

\section{Optical Properties}

Table II summarizes the data for transmittance, refractive index, Abbe's number, and the temperature coefficient of the refractive index at $527 \mathrm{~nm}$ for $\mathrm{TF} 40$ and $\mathrm{TF}$ 
Table II. Optical properties of TF40 and TF70

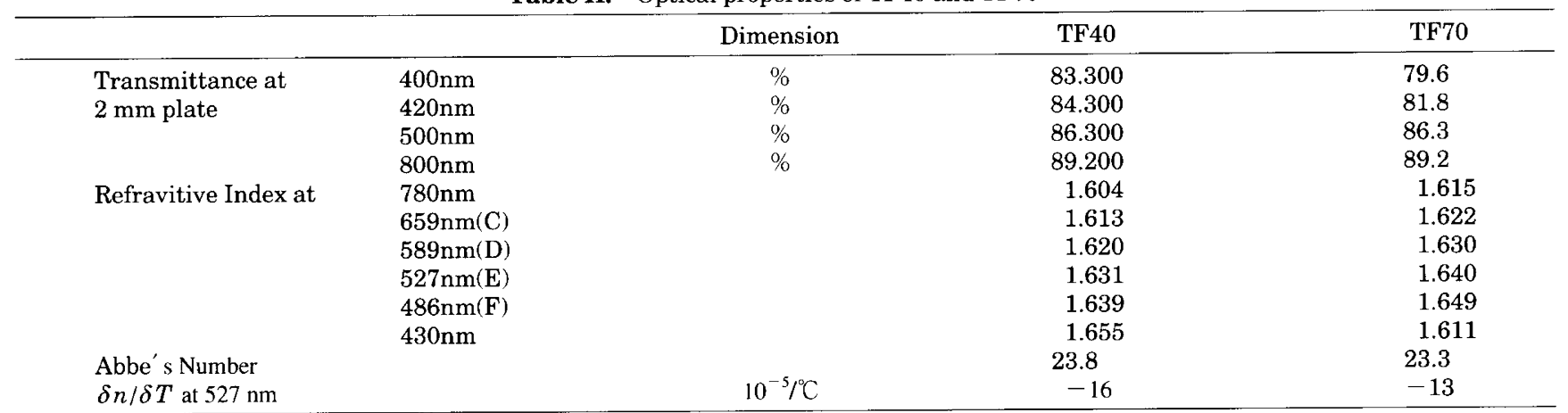

(): Fraunhofer line code.

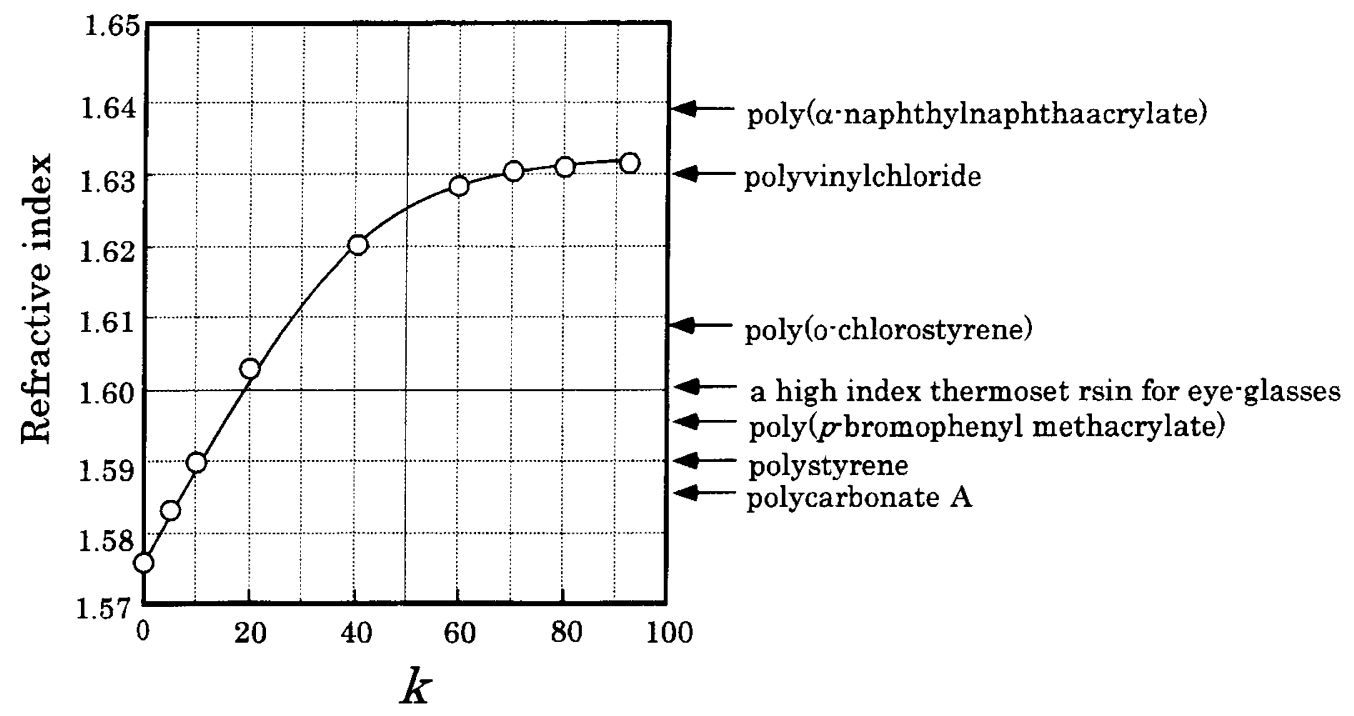

Figure 8. Copolymer composition dependence of the refractive index at the Fraunhofer D line (589 nm) and comparison with other glassy polymers.

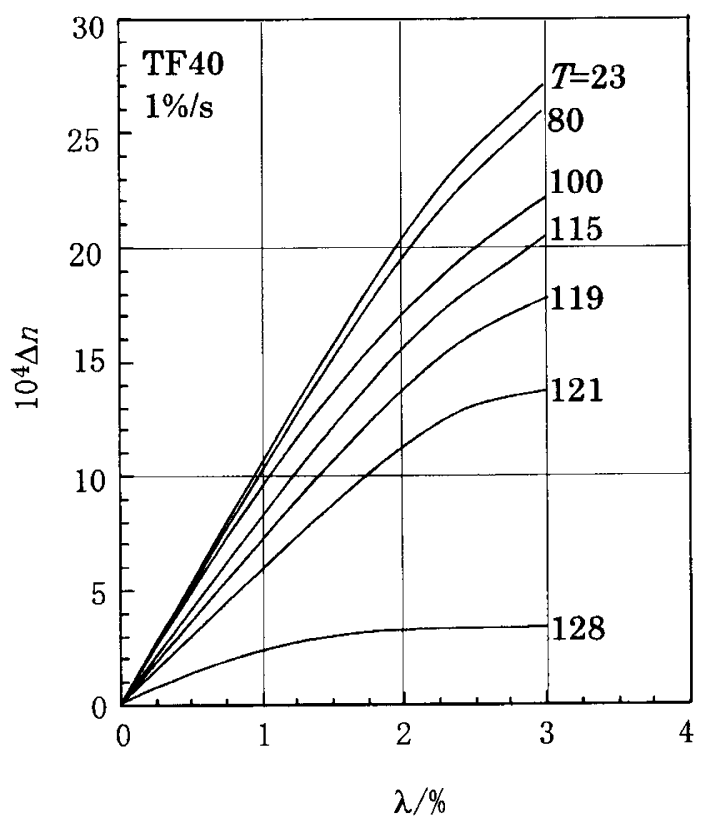

Figure 9. The $\Delta n$ vs. $\lambda$ plots for $\mathrm{TF} 40$ at various temperatures in the range of $23-128^{\circ} \mathrm{C}$.
70. Figure 7 shows a transmittance spectrum for TF40. The spectrum as well as the transmittance values indicate that the samples are visibly clear and transparent. As shown in Table II, the refractive indices are above 1.6 at all wavelengths and higher than those of other polymers used for optical devices. This feature is more clearly understood from Figure 8, where the refractive indices at the Fraunhofer D line $\left(n_{\mathrm{D}}\right)$ plotted against $k$ are compared with those of other amorphous polymers. The value of $n_{\mathrm{D}}$ increases with increasing $k$, exceeds 1.6 at $k>0.2$, and levels off about 1.63 . The last value is comparable with the $n_{\mathrm{D}}$ of harogen-containing polymers. It is remarkable that our polyester with $k>0.1$ has a higher refractive index than polystyrene and bisphenol A polycarbonate which are widely used as a high refractive index thermoplastic. Although these are not thermoplastics, new thermoset resins with $n=1.60$ have been invented for use as eye-glasses. ${ }^{17}$ Our polyester is comparable with those in the magnitude of $n$.

\section{Rhe-optical Proprieties}

Figure 9, which shows the elongation ratio dependence of $\Delta n$ for TF 40 , indicates that the dependence is linear at small $\lambda$ and turns to be upwards-convex at 
Table III. Results from analysis by Fujita's method

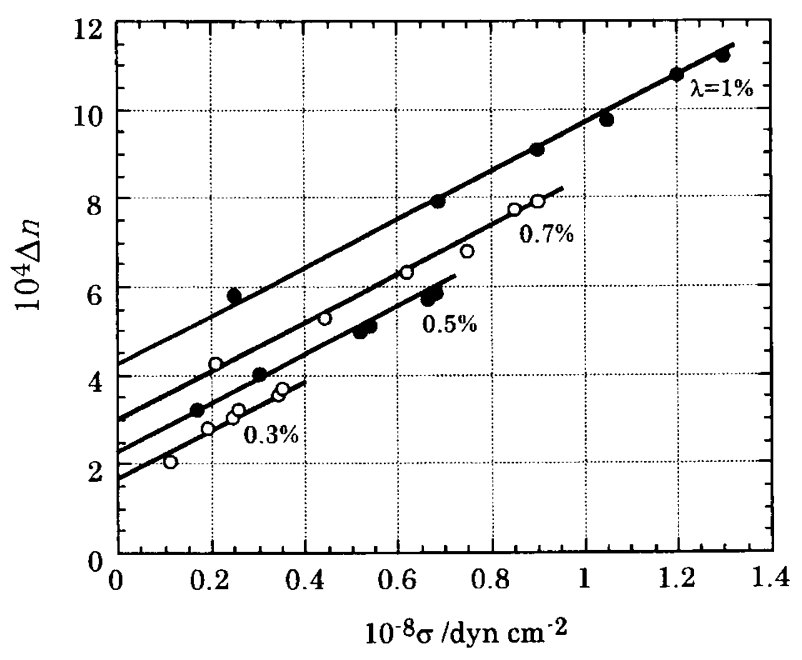

Figure 10. The stress dependence of $\Delta n$ at different elongation ratios for sample TF40.

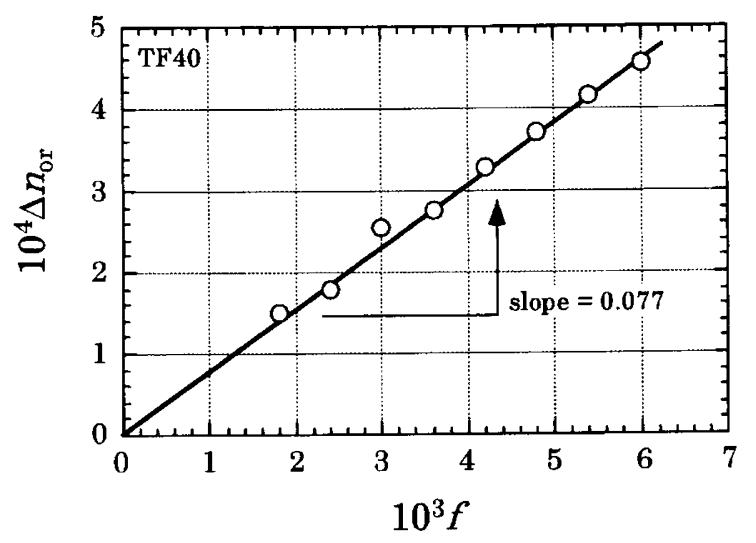

Figure 11. Evaluation of the intrinsic birefringence $\left(\Delta n_{0}\right)$ form the slop of $\Delta n_{\text {or }} v s$. the orientation factor $(f)$.

large $\lambda$, reflecting the yielding of the sample. With increasing the measuring temperature $(T)$, the initial slope decreases and the linear region shrinks. Since this sample's $T_{\mathrm{g}}$ is $124^{\circ} \mathrm{C}$, we see that the linearity between $\Delta n$ and $\lambda$ persists up to $\lambda<1.5 \%$ at $T<T_{\mathrm{g}}$. Although the results are not shown here, it was found that when the elongation rate was increased from 0.1 up to $20 \% \mathrm{~s}^{-1}$, the $\Delta n$ vs. $\lambda$ plot remained unchanged if $\lambda<1 \%$. Since this feature suggests linear elasticity to hold if $\lambda<1 \%$, we calculated the desired set of $\Delta n$ and $\sigma$ data in the range of $\lambda$ from 0.3 to $1 \%$ with an interval of $0.1 \%$. Figure 10 plots $\Delta n$ against $\sigma$ for $\lambda=0.3,0.5,0.7$ and $1 \%$. The straight lines fitting the data points for each $\lambda$ are parallel, showing that the slope does not depend on $\lambda$. The slope and intercept give $C_{\mathrm{e}}$ and $\Delta n_{\mathrm{or}}$, and the latter plotted against $f(=3 / 5 \lambda)$ allows $n_{0}$ to be evaluated as successfully as illustrated in Figure 11. Table III summarizes the values of $\Delta n_{\mathrm{o}}$ and $C_{\mathrm{e}}$ obtained in this way for seven samples examined.

\begin{tabular}{ccc}
\hline Sample & $\Delta n_{\mathrm{o}}$ & $\frac{C_{\mathrm{e}}}{10^{-12} \mathrm{~cm}^{2} \mathrm{dyn}^{-1}}$ \\
& & 5.52 \\
TF10 & 0.161 & 5.23 \\
TF20 & 0.115 & 4.97 \\
TF40 & 0.077 & 4.52 \\
TF60 & 0.072 & 4.21 \\
TF70 & 0.068 & 3.96 \\
TF80 & 0.064 & 3.88 \\
TF95 & 0.058 & \\
\hline
\end{tabular}

\section{DISCUSSION}

According to Crag, ${ }^{18}$ PET has a relatively large $\Delta n_{0}$ (ca. 0.2). Adding the BPEF unit to PET decreases $\Delta n_{0}$ drastically as seen from Table III. This feature indicates the intrinsic birefringence of the BPEF unit to be quite small or even negative, because the observed $\Delta n_{\mathrm{o}}$ for copolymers is the volume average of $\Delta n_{0}$ for the constituting units. Assuming that the specific volumes of the BPEF and DMT units are nearly equal and noticing that the EG unit does not contribute to the birefringence because it has no aromatic rings, we can express $\Delta n_{0}$ of our polyester by

$$
\Delta n_{0}=w_{2} \Delta n_{0} \cdot \mathrm{BPEF}+\left(1-w_{2}\right) \Delta n_{0} \cdot \mathrm{DMT}
$$

where $w_{2}$ is the weight fraction of the BPEF unit defined by $w_{\mathrm{BPEF}} /\left(w_{\mathrm{BPEF}}+w_{\mathrm{DMTA}}\right)$, and $\Delta n_{0} \cdot \mathrm{BPEF}$ and $\Delta n_{0} \cdot \mathrm{DMT}$ are the intrinsic birefringences of the BPEF and DMT units, respectively. Figure 12 plots $\Delta n_{0}$ as a function of $w_{2}$, and shows that the data points can be fitted by a straight line within experimental error. According to eq 5 , the intercept at $w_{2}=0$ provides $\Delta n_{0}$.DMT, and the figure gives 0.22 for $\Delta n_{0}$.DMT, which agrees with the reported one. ${ }^{18}$ The magnitude of $\Delta n_{\mathrm{o}}$.BPEF estimated from the intercept at $w_{2}=1$ is close to zero. Since aromatic rings are optically anisotropic, this small $\Delta n_{0}$.BPEF can be interpreted as due to the four aromatic rings in the BPEF unit spatially arranged so as to cancel their anisotropy each other.

Figure 13 shows the structure for the BPEF unit determined by the energy minimization. The two aromatic rings and the five-member ring of fluorene are in the same plane (plane F) defined by the two tetrahedral bonds of the cardo carbon ( $\mathrm{O}$ in the figure). Against the plane F, the two phenol rings are antisymmetric and the rotational angle against the plane (plane $\mathrm{P}$ ) defined by the two other tetrahedral bonds (OA and $\mathrm{OB}$ ) is $45^{\circ}$. Based on this structure, the intrinsic birefringence of the BPEF unit is calculated to be 0.09 , which is smaller than 0.29 calculated for the DMTA unit by the same method. The difference implies that the stereo structure of the $\mathrm{BPEF}$ unit is responsible for its small birefringence. However, the calculated value is higher than the one estimated from Figure 12, i.e., $\Delta n_{0 \cdot \mathrm{BPEF}}<0.01$.

\section{CONCLUSION}

We examined optical properties of a series of new polyester resins synthesized from BPEF, DMT, and EG and 


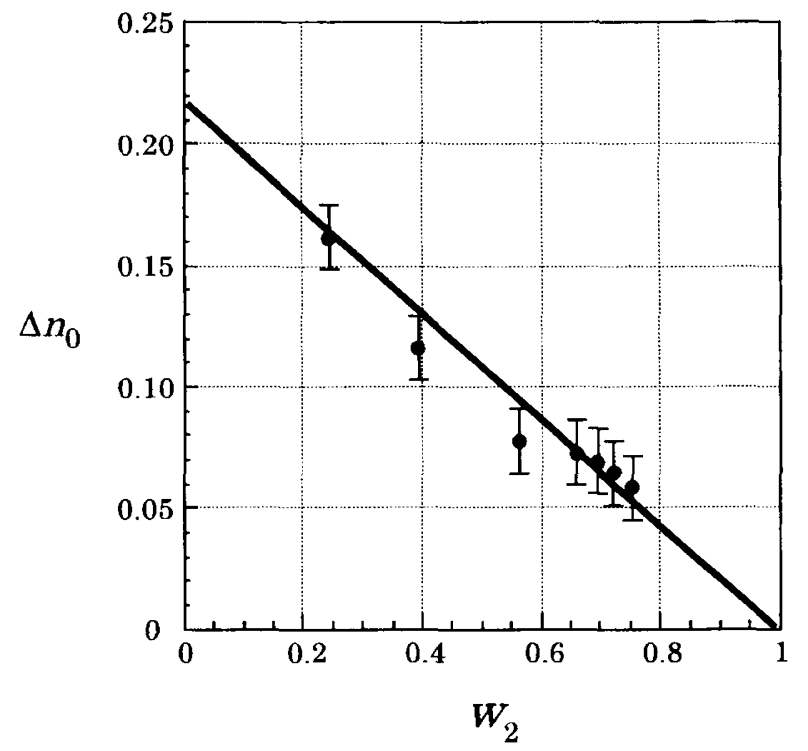

Figure 12. Weight fraction $\left(w_{2}\right)$ dependence of $\Delta n_{0}$, which substantiates eq 5 .

found that their refractive indices were considerably high and their birefringences were low. By using Fujita's method, the intrinsic birefringence of the polyester $\Delta n_{0}$ was evaluated to be about 0.07 for $k=0.7$, which is about one third of the value commonly observed for poly(ethylene terephthalate). Molecular modeling of the BPEF unit indicates that the fluorene side chain is perpendicularly aligned to the main chain and this stereo structure is responsible for the small birefringence of the polyesters.

Acknowledgment. The authors are grateful to $\mathrm{K}$. Yao, K., Igarashi, and T. Kushimoto for their interest in this work and to Prof. H. Saito and Prof. T. Inoue for allowing us to use their rheo-optometer.

\section{REFERENCES}

1. H. Saito, and T. Inoue, J. Polym. Sci., Part B, 25, 1629 (1987).

2. F. Ide, in "Refractive Index Control of Transparent High Polymers" Japan Chemical Society, Ed., 1998, Vol. 39, Chapter 1, p 3.

3. M. Fuji, K. Yao, K., Igarashi, and T. Kushimoto, U. S. Patent 5530086 (1996).

4. D. Brewster, Trans. Roy. Soc. (London), 156, (1816).

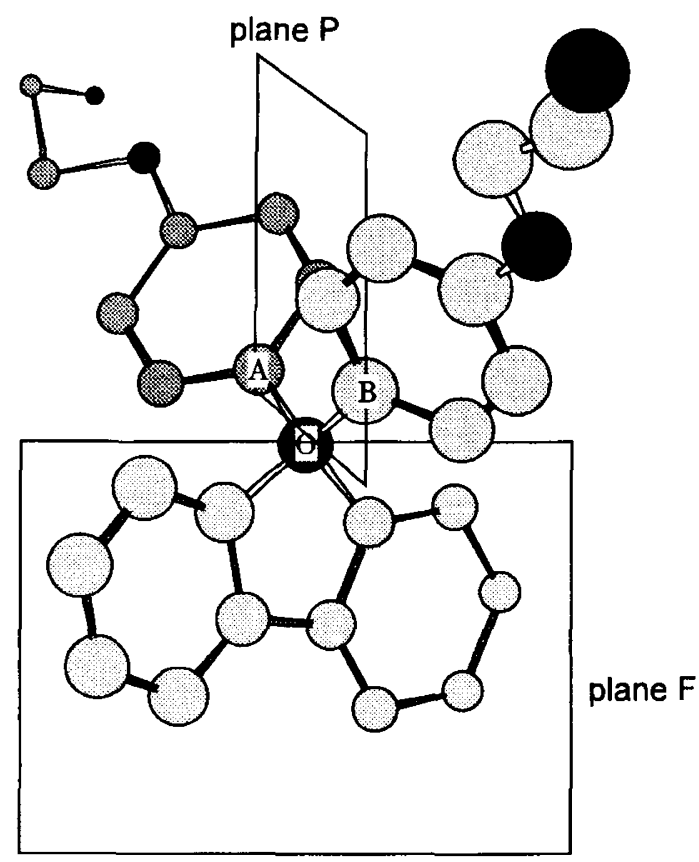

Figure 13. A molecular model for the BPEF unit determined by the energy minimization method.

5. T. Inoue and K Ozaki, Macromolecules, 29, 1595, (1996).

6. A. Fujita, H. Saito, and T. Inoue, Polym. Prepr., Jpn., 41, 4451 (1992).

7. J. D. Ferry, in "Viscoelastic Properties of Polymers" 3rd ed, John Wiley \& Sons, Inc, New York, N. Y., 1980, Chapter 15.

8. S. Matsuoka, "Relaxation Phenomena in Polymers", Hanser Publisher, Munich, 1992.

9. I. M. Ward, J. Polym. Sci., Polym. Symp., 58, 1 (1977).

10. T. Inoue and K Ozaki, Polym. J., 28, 76, (1996).

11. M. Fuji, K. Yao, K. Igarashi, and K. Sakurai, Polym. Prepr., Jpn., 43, 2416 (1994).

12. G. Odian, in "Principles of Polymerization", John Wiley \& Sons, Inc, New York, N. Y., 1993, Chapter 2.

13. M. Yamada, K. Okimi, and M. Nagano, JPS H7-165657 (1995).

14. Y. Aikawa, Kobunshi Ronbunshu, 51, 237, 1994

15. For example see p 209 in "Polymer Handbook" 3rd ed, John Wiley \& Sons, Inc, New York, N. Y., 1989.

16. W. J. MacKnight, F. E. Karasz, and J. R. Fried, in "Polymer Blends" D. R. Paul and S. Newman,. Ed., Academic Press, New York, N. Y., 1978, Chapter 5.

17. H. Kawai, in "Refractive Index Control of Transpearent Polymers" Japan Chemical Society, Ed.,1998, Chapter 18.

18. S. K. Grag, J. Appl. Polym. Sci., 27, 2857, (1982). 